\title{
ORIGINAL ARTICLE \\ The tempo and modes of evolution of reproductive isolation in fungi
}

\author{
T Giraud $^{1}$ and S Gourbière ${ }^{2,3,4}$
}

Reproductive isolation is an essential ingredient of speciation, and much has been learned in recent years about the evolution of reproductive isolation and the genetics of reproductive barriers in animals and plants. Fungi have been neglected on these aspects, despite being tractable model eukaryotes. Here, we used a model fitting approach to look at the importance of different barriers to gene flow to explain the decrease of reproductive compatibility with genetic distance in fungi. We found support for the occurrence of reinforcement in the presyngamy compatibility among basidiomycetes. In contrast, no evidence for reinforcement was detected in ascomycetes, concurring with the idea that host/habitat adaptation in this group can pleiotropically cause reproductive isolation. We found no evidence of a snowballing accumulation of postsyngamic reproductive incompatibilities in either ascomycetes or the complex of anther smut fungi. Together with previous studies, our results suggest that ecologically based barriers to gene flow and karyotypic differences may have an important role in hybrid inviability and sterility in fungi. Interestingly, hybrid sterility appeared to evolve faster than hybrid inviability in fungi. Heredity (2012) 109, 204-214; doi:10.1038/hdy.2012.30; published online 6 June 2012

Keywords: Microbotryum violaceum; Saccharomyces; postzygotic; prezygotic; snowball; sympatry

\section{INTRODUCTION}

Reproductive isolation is an essential determinant of the origin and maintenance of biological diversity. A fundamental measure of the evolution of reproductive isolation is the relationship between the degree of reproductive compatibility and the level of genetic divergence between taxa (Knowlton et al., 1993; Edmans, 2002; Fitzpatrick, 2002). Identifying the shape of this relationship is important in providing key insights into the genetic basis and mechanisms underlying reproductive isolation. This has now been done using comparative data in several animal (Coyne and Orr, 1989; Coyne and Orr, 1997; Sasa et al., 1998; Presgraves, 2002; Bolnick and Near, 2005; Weins et al., 2006) and plant taxa (Widmer et al., 2009).

Fungi are excellent systems to study eukaryotic speciation. They have many experimental advantages (Stajich et al., 2009), such as small genomes, a haploid phase for analyses and controlled experimental crosses, easily scored karyotypes, quantifiable fitness parameters (that is, reproduction, survival), and there exist many complexes of sibling species adapted to different hosts or habitats. Although fungi are highly suitable for investigating isolating barriers, including the role of chromosomal rearrangements and of extrinsic (that is, ecologically based) vs intrinsic (that is, genetic incompatibilities) factors contributing to inviability and sterility, they have just begun to be used in the study of speciation (Delneri et al., 2003; Dettman et al., 2007; Greig, 2007; Lee et al., 2008; Anderson et al., 2010). Investigating speciation in fungi is particularly relevant to the study of fungal pathogens as plant fungal diseases frequently emerge via host-shift speciation (Giraud et al., 2010). Such fungi are responsible for $30 \%$ of all emerging plant diseases (Anderson et al.,
2004), and have tremendous negative impacts on human wellbeing through agricultural and economic losses as well as threats to biodiversity.

Here, we investigated the evolution of reproductive isolation in fungi to infer the relative importance of the different types of isolating barriers in this group. Our general aims were to extend the knowledge we have gained for eukaryotes in animals and plants to fungi and to advance research on the mechanisms of speciation in fungi, a taxonomic group that has long been neglected. We used comparative data present in the literature on the change in the degree of reproductive isolation with genetic distance between fungal species (Le Gac et al., 2007b; Le Gac and Giraud, 2008; de Vienne et al., 2009). Such data were available for the two large phyla of 'higher fungi', ascomycetes and basidiomycetes. These two phyla differ substantially in the reproductive component of their life cycle (see typical sexual life cycles on Figure 1a and b), which has important consequences for mechanisms of speciation (Giraud et al., 2006; Giraud et al., 2010; Gladieux et al., 2011). We also used several data sets dealing with the evolution of hybrid inviability and sterility within a complex of basidiomycete pathogenic fungi causing anther smut disease (Le Gac et al., 2007a). We compared each of these data sets to theoretical models predicting the decrease in reproductive compatibility between species according to different barriers to gene flow. These models predict the temporal changes in compatibility between species according to the rate of substitution between them and the way those substitutions interact (or not) to generate reproductive incompatibilities. Specifically, three models are considered, which respectively yield (i) an accelerating decline of the log of

${ }^{1}$ Laboratoire Ecologie, Systématique et Evolution, UMR8079, CNRS, Univ Paris-Sud 11, Orsay, France; ${ }^{2}$ University of Sussex, School of Life Sciences, Centre for the Study of Evolution, Brighton, UK; ${ }^{3}$ Univ. Perpignan Via Domitia, Ecologie et Evolution des Interactions, Perpignan, France and ${ }^{4} \mathrm{CNRS}$, Ecologie et Evolution des Interactions, Perpignan, France

Correspondence: Dr S Gourbière, Univ. Perpignan Via Domitia, Ecologie et Evolution des Interactions, UMR 5244, F-66860 Perpignan, France.

E-mail: gourbier@univ-perp.fr

Received 14 September 2011; revised 15 March 2012; accepted 11 April 2012; published online 6 June 2012 
a
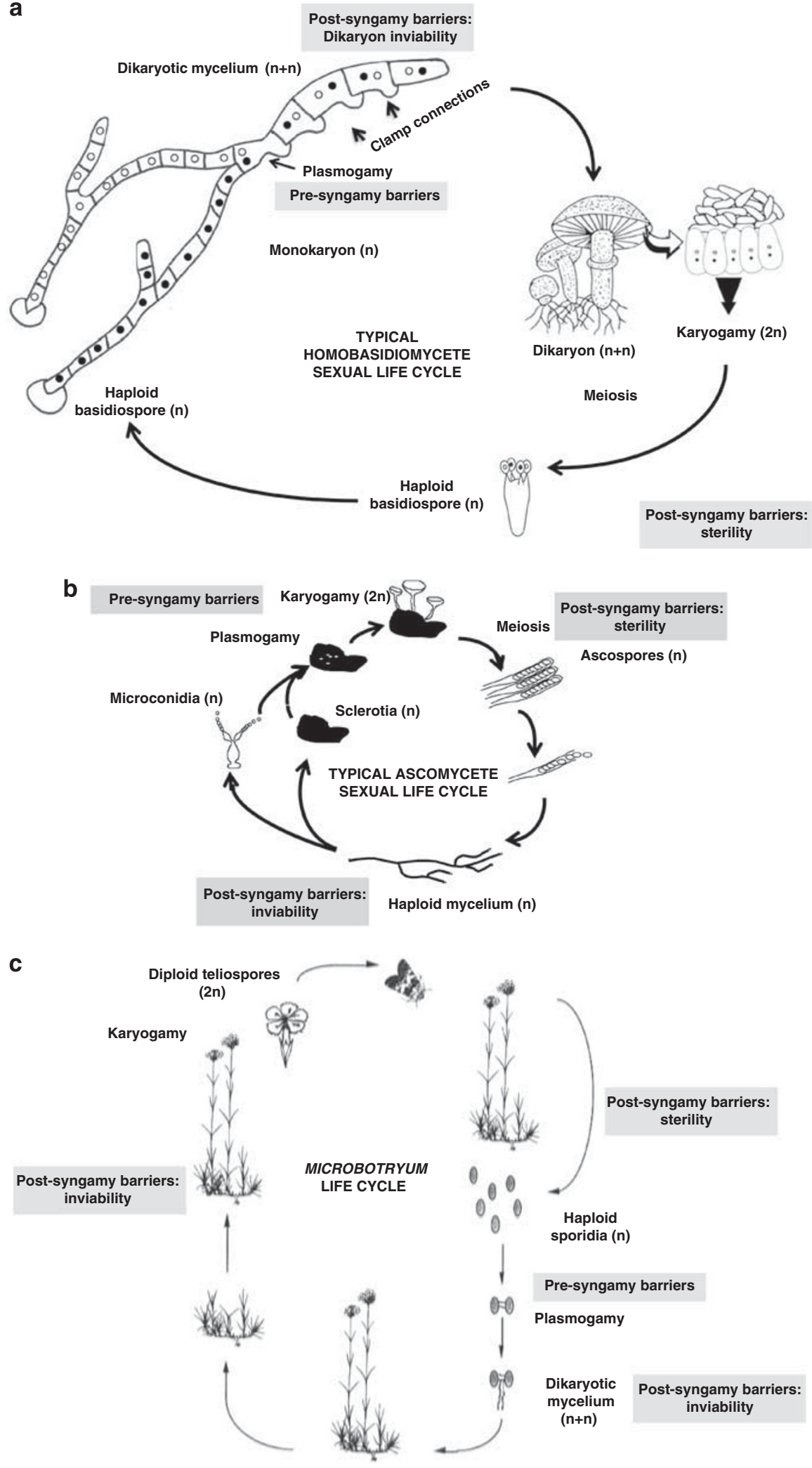

Figure 1 Typical life cycle of homobasidiomycetes (mushrooms, a), of ascomycetes (b) and of Microbotrum (c), with location of presyngamy vs postsyngamy reproductive barriers.

hybrid fitness with increasing genetic distance-a 'snowball' effect (Orr, 1995; Orr and Turelli, 2001), (ii) a decelerating decrease in the log of compatibility-a 'slowdown' effect (Gourbière and Mallet,
2010), or (iii) a constant rate of loss in the log of hybrid fitness-a 'linear' effect (Gourbière and Mallet, 2010). We used a model fitting approach to test which of these models, and underlying mechanisms 
of reproductive isolation, were most likely to explain the decrease of compatibility with genetic distance observed in the different taxa of fungi.

\section{MATERIALS AND METHODS}

\section{Modeling of the evolution of reproductive isolation with genetic distance}

We used three theoretical models that were previously developed to predict the evolution of reproductive isolation as a function of genetic distance between taxa (Gourbière and Mallet, 2010). We refer to the original paper for a complete justification of the modeling assumptions, a formal description of the models, and the mathematical derivation of the expected evolution of reproductive isolation (Gourbière and Mallet, 2010). To produce such predictions one has to model (i) the number of substitutions between two incipient species according to the time since divergence, (ii) the number of incompatibilities resulting from these substitutions, and finally (iii) the level of reproductive compatibility associated with these incompatibilities. From this general principle, three models were derived under separate assumptions on the rate of substitution and on the way substitutions interact to produce incompatibilities.

The first model assumes that substitutions accumulate at a constant rate between diverging lineages, and considers 'Dobzhansky-Muller' (DM) epistatic incompatibilities between pairs of substitutions. The second model also assumes a constant molecular clock, but each substitution can lead to a simple (non-epistatic) incompatibility. The third model combines a decelerating substitution rate and simple (non-epistatic) incompatibilities. In models 1 to 3 , the number of postzygotic incompatibilities then increases with the square of the time since divergence, linearly with time, and slower than linearly with time (Figure 2a). These models are refered to as 'snowball' (Orr, 1995; Orr and Turelli, 2001), 'linear' and 'slowdown' models (Gourbière and Mallet, 2010), respectively. The number of incompatibilities was mapped into reproductive isolation by assuming that every incompatibility has an identical negative effect on reproductive compatibility, and combining these effects multiplicatively (see Gourbière and Mallet (2010) for a discussion of these assumptions). Figure $2 \mathrm{~b}$ shows the typical evolution of the natural logarithm of compatibility expected through time under the three models.

\section{Fitting reproductive compatibility data to the models}

It is worth pointing out that in all the data sets used in this study (see section 'Data sets') each pair of species is represented by a single value (associated genetic distance and reproductive isolation), although there is likely to be variation in both values as multiple isolates were used to generate each of these point estimates. This heterogeneity in the data, coupled with the non-independence of comparisons of the same or related species, makes the use of a complete likelihood fitting approach difficult (See Appendix C in Gourbière and Mallet (2010) for a discussion of the difficulties associated with the statistical handling of this type of data set). The three models were thus fitted by minimizing the sums of square of the differences between observed and expected values of log-transformed compatibilities. According to the above assumptions, the expectations under the linear, snowball and slowdown models are given by $E\left(\ln C_{\mathrm{T}}\right)=e_{1} \mathrm{~T}, E\left(\ln C_{\mathrm{T}}\right)=e_{2} \mathrm{~T}^{2}$ and $E\left(\ln C_{\mathrm{T}}\right)=e_{3} \ln (1+$ aT) $/ a$, and we refer to the previous article for a definition of parameters $e_{1}, e_{2}$ and $e_{3}$ as functions of the rate of substitution, the probability for a pair of substitutions to form an incompatibility, and the deleterious effect of any given incompatibility (Gourbière and Mallet, 2010). The parameter $a$ measures the rate of deceleration of the substitution rate in the slowdown model. For most data sets, the measure of compatibility is continuous and was thus fitted directly to the above expectations. For discrete postsyngamy compatibility data we produced discrete predictions from the above formulae by using the method of discretization developed by Gourbière and Mallet (2010). After the best fit produced by each model was identified, we estimated the goodness of fit by a standard analysis of residuals using non-parametric tests following Gourbière and Mallet (2010). A Kruskal-Wallis test was performed to check whether the model overestimated the data at low genetic distances and underestimated them at large genetic distances, or vice-versa. A test of autocorrelation was further performed on continuous data to detect any excess of positive or negative residuals at more intermediate genetic distances. When analyzing discrete compatibility data, the value of residuals can be null. Multiple Wilcoxon-Mann-Whitney tests were then used to compare the mean ranks of negative vs positive residuals, as well as the mean ranks of null vs negative and null vs positive residuals. Overall, the quality of the fit of models to each data set was thus evaluated both by a quantitative measure of the differences between predictions and observations (sum of squares produced by the three models were compared through basic F statistics), and by a qualitative assessment of whether the models provided good predictions at all genetic distances (using the $P$-values of the non-parametric tests).

\section{Terminology and life cycles}

Prezygotic and postzygotic isolations are typically distinguished in the speciation literature because they correspond to different, critical steps with respect to parental investment in the progeny (the 'zygote'). The terms may not be appropriate in basidiomycete fungi (for example, mushrooms, smuts and rusts), because of their extended dikaryotic stage, which in fact represents the main step of their life cycle. In basidiomycetes, the nuclear fusion can occur much later than syngamy (that is, fertilization), so that the distinction prezygotic vs postzygotic is not the most relevant. Actually, the important distinction to be
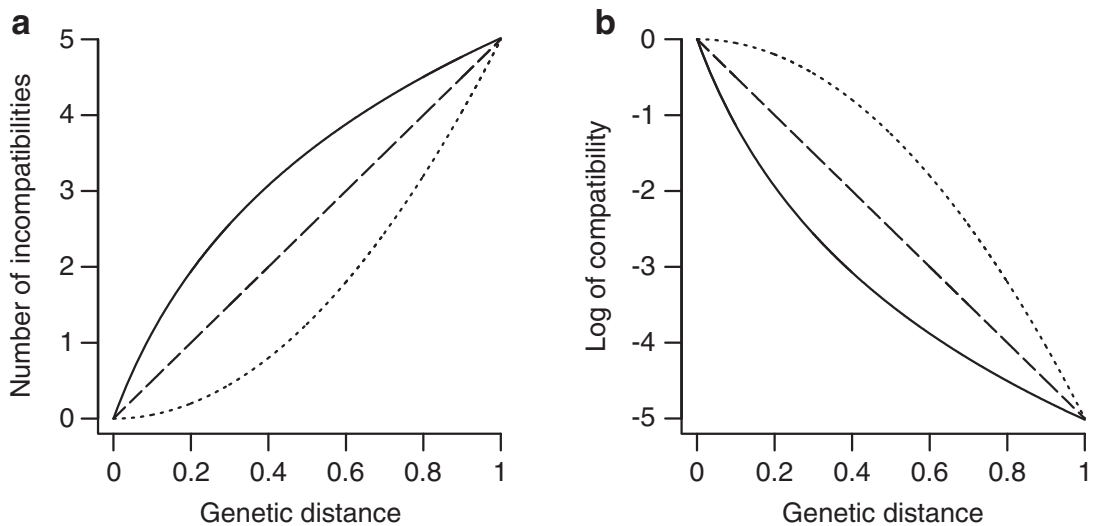

Figure 2 Theoretical models of the evolution of reproductive isolation. Three models were derived according to different assumptions about the rate of substitution and the way substitutions interact to produce incompatibilities between taxa. The 'snowball' model (constant substitution rate and epistatic interaction), the 'linear' model (constant substitution rate and non-epistatic interaction) and the 'slowdown' model (decelerating rate of substitution and non-epistatic interaction). (a, b) show the accumulation of incompatibilities and the decline in the logarithm of reproductive compatibility between taxa obtained from these models. Continuous, broken and dotted lines stand for the 'slowdown', 'linear' and 'snowball' model, respectively. 
made for reproductive isolation when considering parental investment is presyngamy and postsyngamy (which in fact correspond to prezygotic and postzygotic stage in most organisms, but not in basidiomycetes). We will therefore hereafter refer to 'presyngamy and postsyngamy' stages for basidiomycetes as well as for ascomycetes, though for ascomycetes the terms postzygotic and prezygotic could be applied. The Figure 1 illustrates typical sexual life cycles for ascomycetes, homobasidiomycetes and Microbotryum.

\section{Data sets}

Basidiomycetes-allopatric and sympatric species. These two data sets on the evolution of reproductive isolation in basidiomycete fungi were collected from a survey of the literature by Le Gac and Giraud (2008) on the existence of sibling species in fungi and on the degree and nature of their reproductive isolation. The two data sets describe the degree of isolation at the presyngamy level among closely related species of basidiomycete fungi occurring in sympatry and allopatry. The degree of reproductive isolation was calculated as $1-p$, where $p$ stands for the proportion of crosses showing evidence of plasmogamy initiation. Plasmogamy is a stage of basidiomycete sexual reproduction whereby the cytoplasms of two parental mycelia fuse together without a fusion of their nuclei, that is, it corresponds to syngamy (Figure 1a). The initiation of plasmogamy was detected by the observation of clamp connections in Homobasidiomycetes and by the observation of conjugations in Microbotryum, the only species complex studied outside of Homobasidiomycetes. A quantitative measure of reproductive isolation was scored, as the proportion of pairs of individuals who did not initiate syngamy. This quantitative measure of the degree of presyngamy reproductive isolation is very similar to the measure of prezygotic isolation used by Coyne and Orr (1989). Genetic distances between species pairs were computed using ITS sequences available in GenBank and the Kimura-2-parameter model.

Ascomycetes-allopatric and sympatric species. These two data sets describe the evolution of reproductive isolation between closely related species of ascomycete fungi (for example, yeasts, moulds) and were also collected in the survey by Le Gac and Giraud (2008) on the existence of sibling species in fungi and on the degree and nature of their reproductive isolation. Quantitative results on the success of crosses between ascomycetes are usually not reported in the literature. The success of in vitro crosses is typically assessed by mixing male and female gametes in petri dishes and by subsequently scoring whether some asci and ascospores develop or not. We therefore considered reproductive isolation in ascomycetes as a discrete measure where $1,0.75,0.50,0.25$ and 0 indicate, respectively, lack of ascus formation, asci without ascospores, abnormal ascospores, viable but sterile ascospores and fertile ascospores (Figure 1b). Presyngamy reproductive isolation is thus indicated by a reproductive isolation equal to 1 . The other categories $(0,0.25,0.50$ and $0.75)$ refer to different levels of postsyngamy isolation. Unfortunately, the data sets were too small for attempting to fit curves separately on presyngamy and postsyngamy data. Genetic distances between species pairs were computed using ITS sequences available in GenBank and the Kimura-2-parameter model.

Microbotryum. The basidiomycete fungal species complex Microbotryum is responsible for anther smut, a naturally occurring venereal disease of many insect-pollinated perennial plant species in the Caryophyllaceae (Hood et al., 2010). Diploid teliospores of Microbotryum are produced in the anthers of infected plants, thereby replacing the pollen (Figure 1c). Deposited on a new host, teliospores undergo meiosis, leading to the production of haploid yeastlike sporidia of opposite mating types. These sporidia can be considered as the gametes of the fungus. Conjugation (syngamy) between sporidia of opposite mating types is required to initiate growth of dikaryotic infectious hyphae. The genus Microbotryum contains several sibling species, each specialized on one or a few plant species (Le Gac et al., 2007a; Refrégier et al., 2008). The genetic distances were calculated using the 700-bp sequence of the gamma-tubulin nuclear gene (Le Gac et al., 2007b). A pairwise distance matrix was obtained using the Kimura two-parameter model.

Microbotryum assortative mating and F1 hybrid inviability. A first data set on assortative mating (that is, isolation at the stage of syngamy) has been obtained by Le Gac et al. (2007b), by mixing in water in ELISA plates sporidia of one mating type from a first Microbotryum species with sporidia of the opposite mating type from a second Microbotryum species, and by then observing under the microscope the percentage of conjugating sporidia (that is, the percentage of gametes undergoing syngamy). The lower the percentage of conjugation, the higher the degree of presyngamy isolation. A second data set on reproductive isolation among anther smut fungi deals with hybrid inviability at the stage of early hyphal growth, after syngamy. This data set has also been obtained by Le Gac et al. (2007b), by plating on agar medium with alpha-tocopherol (promoting hyphal growth) sporidia of one mating type from a first Microbotryum species with sporidia of the opposite mating type from a second Microbotryum species, and by then observing under the microscope the percentage of conjugating pairs growing dikaryotic hyphae. This data set thus regards the first stages of hybrid viability after syngamy. A third and fourth data set on reproductive isolation between Microbotryum species measured hybrid inviability as the ability of hybrids to infect the plants and produce diploid spores. These data have also been obtained by Le Gac et al. (2007b), by plating on agar medium sporidia of one mating type from a first Microbotryum species with sporidia of the opposite mating type from a second Microbotryum species, together with seeds of one parental host plant. Two subdata sets were generated by Le Gac et al. (2007b), by crossing either the species Microbotryum lychnidis-dioicae (specialized on the plant Silene latifolia) with a set of seven other Microbotryum species and then inoculating the hybrids on the plant S. latifolia ('Sl infection data set') or by crossing the species M. silenes-dioicae (specialized on the plant $S$. dioica) with a set of seven other Microbotryum species and then inoculating the hybrids on the plant S. dioica ('Sd infection data set'). The plants were then transferred in the greenhouse to let them flower and to record whether the hybrids could produce symptoms (that is, smutted anthers) in the flowers. This data set thus integrates hybrid viability over the whole life cycle.

Microbotryum F1 hybrid sterility. This data set, obtained by de Vienne et al. (2009), represents the degree of sterility of the viable gametes of the Microbotryum F1 produced by Le Gac et al. (2007b). Diploid spores of F1 hybrids were plated on a nutritive medium where they underwent meioses. Viable sporidia were tested for their ability to conjugate (that is, undergo syngamy) with stock parental sporidia of either mating type and the percentage of conjugating sporidia was recorded.

Microbotryum DNA content in F1 hybrids' gametes. This data set, obtained by de Vienne et al. (2009), represents a measure of the excess of DNA content in the gametes compared with non-hybrid gametes. DNA content of hybrid gametes was estimated using flow cytometry and was normalized by the mean content of non-hybrid gametes.

Microbotryum F2 hybrid inviability. This data set, again obtained by de Vienne et al. (2009), represents the inability of Microbotryum F2 hybrids to infect host plants and produce symptoms. The Microbotryum F1 hybrids produced by Le Gac et al. (2007b) had been allowed to self on germinating plants on agar plates and symptoms were recorded in the greenhouse where the plants had been transferred.

\section{RESULTS}

The results and tests of the quality of the fit of three proposed models to the various data sets are provided in Table 1 (for Basidiomycetes) and in Table 2 (for Ascomycetes).

\section{Basidiomycetes}

The two data sets on the evolution of reproductive isolation in basidiomycete fungi (mushrooms and smuts) describe the degree of isolation at the presyngamy level among closely related species, with species pairs either in sympatry or in allopatry.

Basidiomycetes - allopatric species. The slowdown model provides a better fit to presyngamy isolation data than the snowball model, as indicated by a significantly larger sum of squares $(\mathrm{F}=2.51$, $P=0.001)$. This is due to an observed decrease in reproductive 
Table 1 Evolution of presyngamic and postsyngamic reproductive compatibility with genetic distance in basidiomycetes

\begin{tabular}{ccccc}
\hline Model fitted & $\begin{array}{c}\text { Sum of } \\
\text { squares }\end{array}$ & Kruskall- & Runs & $\mathrm{e}_{1}, \mathrm{e}_{2}$ or $\mathrm{e}_{3} \quad$ a \\
& test & & \\
\hline
\end{tabular}

\begin{tabular}{lccccc}
\hline Basidiomycetes (allopatric), & plasmogamy & initiation vs ITS divergence, $\mathrm{N}=47$ \\
Snowball & 316.6 & 0.465 & 0.008 & -2934.7 & \\
Linear & 201.9 & 0.243 & 0.002 & -127.7 & \\
Slowdown & $\mathbf{1 2 3 . 1}$ & $\mathbf{0 . 6 8 5}$ & $\mathbf{0 . 1 0 0}$ & $-\mathbf{6 6 6 6 . 4}$ & $\mathbf{1 3 0 4 0 . 2}$
\end{tabular}

Basidiomycetes (sympatric), plasmogamy initiation vs ITS divergence, $\mathrm{N}=57$

$\begin{array}{lllll}\text { Snowball } & 622.8 & 2.10^{-4} & 9.10^{-7} & -4405.7\end{array}$

Linear $\quad 391.8 \quad 2.10^{-10} \quad 2.10^{-8} \quad-176.9$

$\begin{array}{lllll}\text { Slowdown } & 139.4 & 0.039 & 0.489 & -2.10^{18}\end{array}$

$2.10^{19}$

Microbotryum, conjugation of sporidia vs g-tubulin gene divergence, $\mathrm{N}=9$

\begin{tabular}{lrrrrr} 
Snowball & 20.5 & 0.023 & 0.048 & -679.6 \\
Linear & 13.5 & 0.023 & 0.048 & -42.7 & \\
Slowdown & $\mathbf{1 . 1}$ & $\mathbf{0 . 0 6 3}$ & $\mathbf{0 . 5 2 4}$ & $-\mathbf{1 . 1 0 ^ { 1 7 }}$ & $\mathbf{2 . 1 0}$ \\
\multicolumn{7}{c}{ Microbotryum, hyphal growth } & of $F 1$ hybrids & vs g-tubulin gene divergence, N $=9$ \\
Snowball & 4.0 & 0.730 & 0.429 & -590.7 \\
Linear & $\mathbf{4 . 8}$ & $\mathbf{0 . 1 9 0}$ & $\mathbf{0 . 5 2 4}$ & $\mathbf{- 3 1 . 6}$ & \\
Slowdown & 3.8 & 0.190 & 0.429 & -13.9 & -15.8
\end{tabular}

Microbotryum, F1 infection on Silene latifolia vs g-tubulin gene divergence, $\mathrm{N}=10$

$\begin{array}{lrrrrr}\text { Snowball } & 23.3 & 0.067 & 0.905 & -987.6 & \\ \text { Linear } & 10.5 & 0.007 & 0.850 & -64.7 & \\ \text { Slowdown } & \mathbf{3 . 3} & \mathbf{0 . 3 8 3} & \mathbf{0 . 8 0 9} & \mathbf{- 1 9 8 5 . 9} & \mathbf{2 8 3 6 . 0}\end{array}$

Microbotryum, F1 infection on Silene dioicae vs g-tubulin gene divergence, $\mathrm{N}=10$

$\begin{array}{lrrrrr}\text { Snowball } & 41.8 & 0.200 & 0.400 & -1146.6 & \\ \text { Linear } & 20.1 & 0.067 & 0.566 & -76.2 & \\ \text { Slowdown } & \mathbf{3 . 4} & \mathbf{0 . 9 8 5} & \mathbf{0 . 0 4 7} & \mathbf{- 8 7 7 3 . 0} & \mathbf{1 4 0 9 5 . 8}\end{array}$

Microbotryum, conjugation of $F 1$ sporidia vs g-tubulin gene divergence, $\mathrm{N}=14$

$\begin{array}{lrrrrr}\text { Snowball } & 10.1 & 0.190 & 0.846 & -530.4 & \\ \text { Linear } & 8.0 & 0.088 & 0.980 & -29.6 & \\ \text { Slowdown } & \mathbf{7 . 7} & \mathbf{0 . 0 8 8} & \mathbf{0 . 8 4 6} & \mathbf{- 4 5 . 5} & \mathbf{2 7 . 4}\end{array}$

Microbotryum, DNA content of F1 hybrid gametes vs g-tubulin gene divergence, $\mathrm{N}=14$

$\begin{array}{llllr}\text { Snowball } & 7.1 & 0.898 & 0.078 & -609.5 \\ \text { Linear } & 4.3 & 0.852 & 0.452 & -33.9 \\ \text { Slowdown } & 4.0 & 0.852 & 0.452 & -54.9\end{array}$

Microbotryum F2 infection on host plants vs g-tubulin gene divergence, $\mathrm{N}=15$

$\begin{array}{lrrrr}\text { Snowball } & 22.1 & 0.448 & 0.259 & -1147.1 \\ \text { Linear } & 7.5 & \mathbf{0 . 5 9 4} & \mathbf{0 . 6 4 3} & -64.6\end{array}$

$\begin{array}{lllll}\text { Slowdown } & 3.8 & 0.779 & 0.970 & -170.7\end{array}$

The first two data sets are measures of presyngamy between pairs of different species of basidiomycetes, whereas the last seven data sets are measures of presyngamy (conjugation of sporidia) and postsyngamy (all remaining data sets) within the microbotryum species complex, which belongs to basidiomycetes. For each data set, the retained model appears in bold (see main text for details on model selection, and Figures 3,5 and 6 for the fits of these modee to ther details on model selection, and Figures 3, 5 and 6 for the fits of these models to the data sets).

compatibility that is much faster than expected under the snowball model. Correlatively, the linear model also does a better job than the snowball $(\mathrm{F}=1.53, P=0.076)$ and the slowdown performs better than the linear model $(\mathrm{F}=1.60, P=0.057)$, although none of these differences reach statistical significance. In addition, only the fit to the slowdown model leads to residuals with no significant autocorrelation in the runs tests $(P=0.100$ vs $P=0.002$ and 0.008 for the linear and snowball models, respectively). The superiority of the slowdown model is consistent with the highly positive estimate of the parameter $a$, which leads to a much more rapid initial decrease in compatibility at low genetic distance than under the snowball and linear models (Figure 3a).

Basidiomycetes - sympatric species. The slowdown model fits this data set much better than the snowball and the linear models. Indeed, there is a large difference in the goodness of fit between the slowdown model and both the snowball $(\mathrm{F}=4.38, P<0.001)$ and the linear $(\mathrm{F}=2.76, P<0.001)$ model. The last two models produce residuals with strong autocorrelation (all $P$-values being $<10^{-5}$ in the analysis of their residuals), as they unambiguously overestimate reproductive compatibility at low genetic distances (Figure 3b). This corresponds to an even faster decrease in reproductive compatibility with genetic distance than observed in allopatry (Figure 3a), which is consistent with a larger estimate of parameter $a$ than obtained with allopatric species.

\section{Ascomycetes}

These two data sets describe the evolution of reproductive isolation between closely related species of ascomycete fungi (yeasts, moulds), occurring, respectively, in sympatry or in allopatry. Increasing isolation corresponds to earlier stages of the barriers to gene flow in the life cycle, from postsyngamy to presyngamy stages.

Ascomycetes-allopatric species. All three models provide similar fit to the data (Figure 4a) and, despite slightly lower sums of squares, neither the snowball $(\mathrm{F}=0.88, P=0.602)$ nor the slowdown $(\mathrm{F}=1.15, P=0.380)$ provides a better fit than the linear model. Analysis of the residuals confirmed the absence of support for any of the models over the others as $P$-values obtained for the three models were roughly similar for each of the performed tests. In other words, we cannot reject the null hypothesis of a simple exponential decrease in compatibility with genetic distance.

Ascomycetes - sympatric species. There is again no support for one of the three models over the others. Although we obtained slightly lower $P$-values in the F-tests between the linear model and either the snowball $(\mathrm{F}=1.09, P=0.351)$ or the slowdown $(\mathrm{F}=1.10, P=0.338)$ models, none of them provide evidence for a departure from the simplest (linear) model (Figure $4 \mathrm{~b}$ ). Analysis of the residuals provides a lower support for the slowdown model $(P=0.006)$ and gives similar outcomes for both the snowball and linear models (none of their distribution of residuals showing a significant departure from random distribution, $P=0.947$ and $P=0.148$ ). There is thus again no support for any of the models over the others.

\section{Microbotryum}

Microbotryum assortative mating. For the anther smut fungi, the slowdown model provides a better fit to this presyngamy isolation data set than the snowball $(\mathrm{F}=16.3, P<0.001)$ and the linear $(\mathrm{F}=10.7, P=0.002)$ models. In addition to this significantly lower sum of squares, the analysis of the residuals shows that it is the only model whose fit provides residuals that are randomly distributed $(P=0.063$ and $P=0.524$ in the two non-parametric tests). The degree of syngamy compatibility goes down rapidly at low genetic distances and ceases to fall afterwards (Figure 5a).

Microbotryum F1 hybrid inviability - hyphal growth ability. Regarding the data set on early hybrid inviability of Microbotryum measured as hyphal growth in vitro (Figure $5 \mathrm{~b}$ ), the fits obtained with the three 
Table 2 Evolution of presyngamic and postsyngamic reproductive compatibility in ascomycetes

\begin{tabular}{|c|c|c|c|c|c|c|c|}
\hline \multirow{2}{*}{ Model fitted } & \multirow{2}{*}{ Sum of squares } & \multirow{2}{*}{ Kruskall-Wallis } & \multicolumn{3}{|c|}{ Wilcoxon-Mann-Whitney } & \multirow{2}{*}{$\mathrm{e}_{1}, \mathrm{e}_{2}$, or $\mathrm{e}_{3}$} & \multirow{2}{*}{ a } \\
\hline & & & $(0,+)$ & $(0,-)$ & $(+,-)$ & & \\
\hline \multicolumn{8}{|c|}{ Ascomycetes (allopatric), spores formation in crosses vs ITS divergence, $\mathrm{N}=20$} \\
\hline Snowball & 26.4 & 0.9474 & 0.9474 & - & - & -1842 & \\
\hline Linear & 28.2 & 0.1482 & - & - & 0.1482 & -92.5 & \\
\hline Slowdown & 23.1 & 0.0056 & - & - & 0.0056 & -200.1 & 40.5 \\
\hline \multicolumn{8}{|c|}{ Ascomycetes (sympatric), spores formation in crosses vs ITS divergence, $\mathrm{N}=69$} \\
\hline Snowball & 208.4 & $4.10-5$ & 0.2967 & 0.0125 & $1.10-6$ & -3080.7 & \\
\hline Linear & 232.2 & $1.10-7$ & 0.4134 & 0.0001 & $1.10-8$ & -115.2 & \\
\hline Slowdown & 185.4 & $1.10-4$ & 0.2195 & 0.0796 & $1.10-6$ & -196.0 & 34.1 \\
\hline
\end{tabular}

Spore formation was scored by a discrete variable and model predictions were produced for the same variable. Some differences between observed and predicted compatibility between allopatric species happened to be null, which lead us to test for the randomness of the distribution of 0 vs positive and 0 vs negative residuals, in addition to the usual test on positive vs negative residuals. For each data set, the retained model appears in bold (see main text for details on model selection, and Figure 4 for the fits of these models to the data sets).
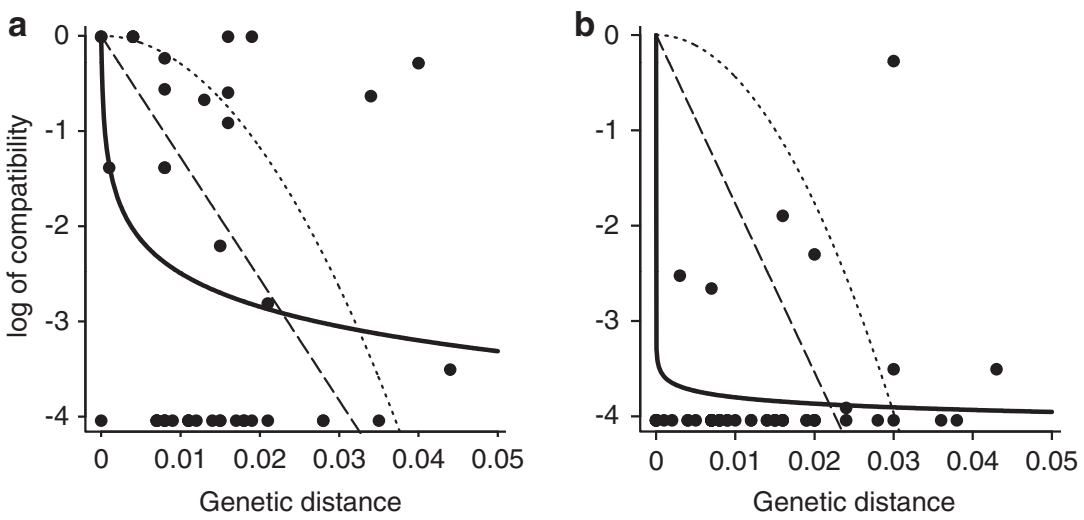

Figure 3 Evolution of reproductive isolation in Basidiomycetes. (a, b) give the evolution of presyngamic isolation in allopatry and sympatry, respectively. Continuous, broken and dotted lines stand for the 'slowdown', 'linear' and 'snowball' model, respectively. For each data set, the best model appears in bold.
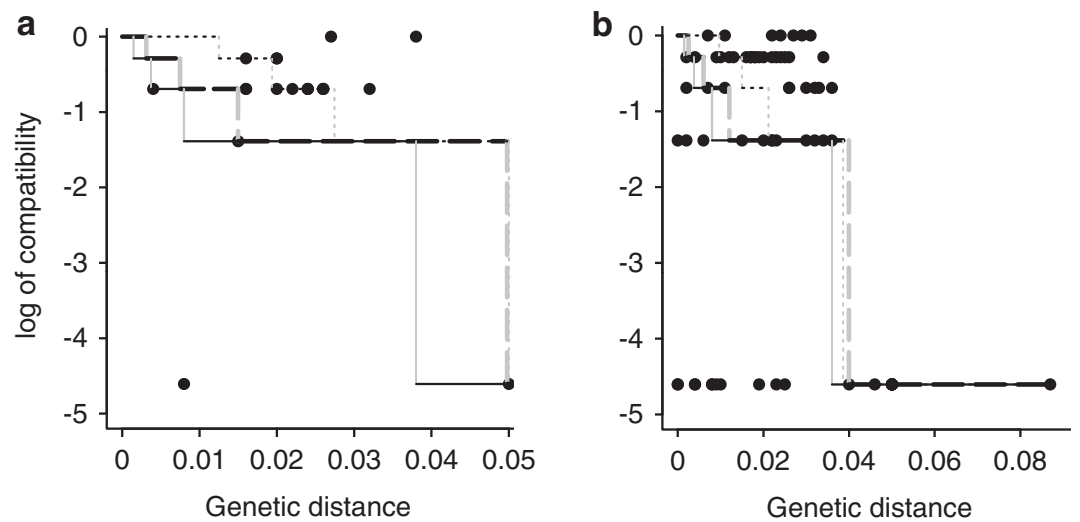

Figure 4 Evolution of reproductive isolation in Ascomycetes. $\mathbf{a}$ and $\mathbf{b}$ show the evolution of presyngamic and postsyngamic isolation in allopatry and sympatry, respectively. Continuous, broken and dotted lines stand for the 'slowdown', 'linear' and 'snowball' model, respectively. For each data set, the best model appears in bold. As data were made of discrete measures of reproductive isolation, model predictions were transformed into a discrete variable (see main text for details).

models are all good, as evidenced by the low sums of squares and the absence of departure from a random distribution of their residuals (all $P$-values in the analysis of residuals are $>0.190$ ). Neither the snowball $(\mathrm{F}=0.73, \quad P=0.665)$ nor the slowdown $(\mathrm{F}=1.07$,
$P=0.467)$ model received a stronger support from the data than the linear model did. However, we must point out that the slowdown model fits the data only for a negative value of the parameter $a$, which actually produces a slightly convex snowball-like relationship. 

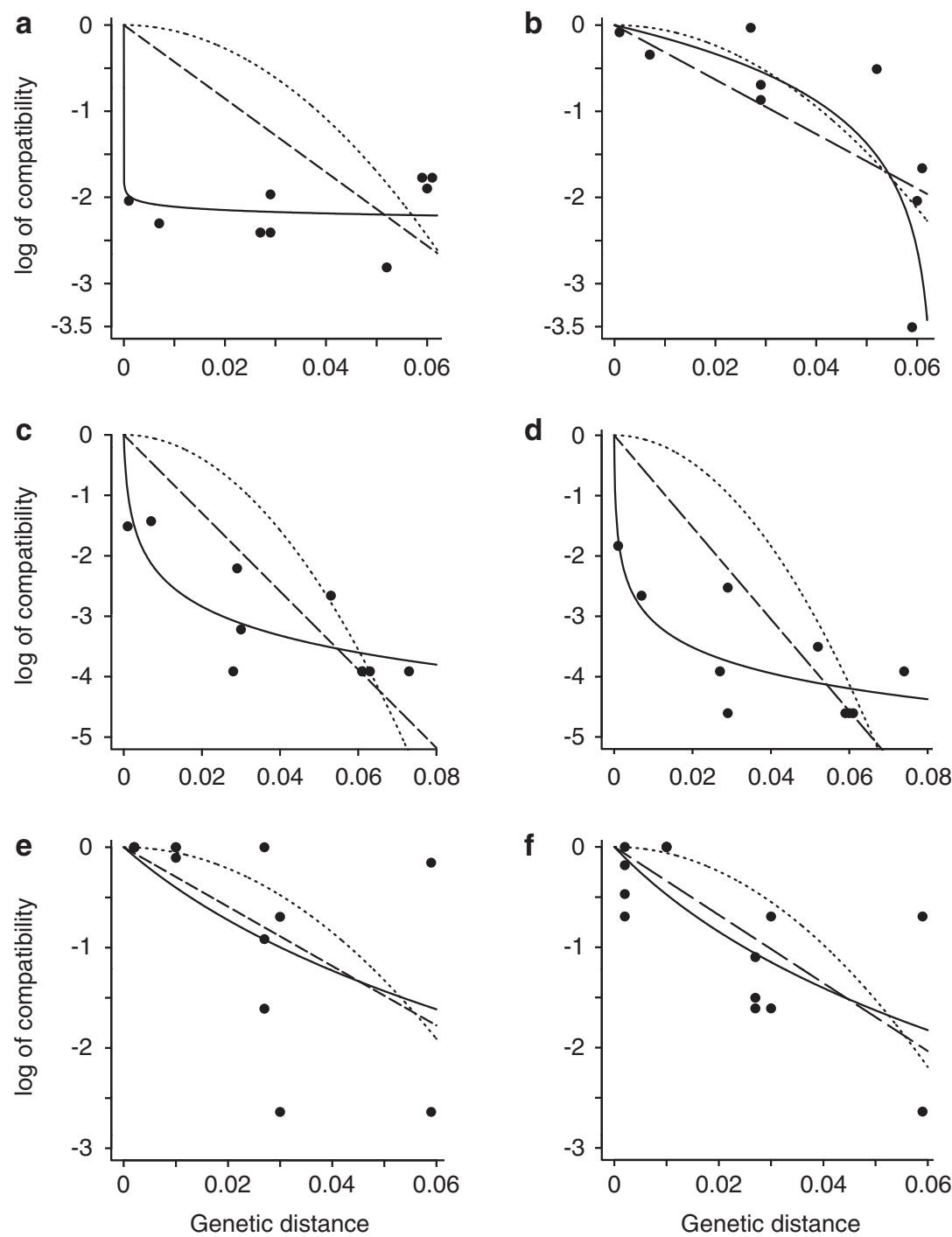

Figure 5 Evolution of reproductive isolation in Microbotryum (Basidiomycete). (a, b) show the evolution of assortative mating between sporidia, and hybrid inviability during F1 hybrids' hyphal growth, respectively. (c, d) describe F1 hybrid inviability as measured by the inability of hybrids to infect plants and produce diploid spores on S. latifolia and $S$. dioica, respectively. $(\mathbf{e}, \mathbf{f})$ show the evolution of F1 hybrids' gamete sterility and the evolution of DNA content in F1 hybrids' gametes, respectively. Continuous, broken and dotted lines stand for the 'slowdown', 'linear' and 'snowball' model, respectively. For each data set, the best model appears in bold.

Microbotryum F1 hybrid inviability — hybrid infectivity. Regarding the evolution of the ability of Microbotryum hybrids to produce symptoms on S. latifolia, the slowdown model fits the data better than the snowball model $(\mathrm{F}=3.82, P=0.036)$ because of a failure of reproductive compatibility at low genetic distances that is earlier than expected under the hypothesis of accumulation of epistatic incompatibilities (Figure 5c). The linear model then also seems to provide a better fit than the snowball model, but the difference fails statistical significance $(\mathrm{F}=1.97, P=0.175)$. Similarly, the difference between the fits of the slowdown and the linear model does not reach standard statistical threshold $(P=2.79, P=0.081)$. The support for the slowdown model is nevertheless consistent with the high positive estimate of the parameter $a$, which allows for the rapid observed decrease in reproductive compatibility, and by substantially larger $P$-values in the analysis of the residuals ( $P=0.383$ vs 0.067 and 0.007 in the test of the mean position of negative and positive residuals). Regarding the evolution of the ability of Microbotryum hybrids to produce symptoms on S. dioica (Figure 5d), the slowdown model received an even stronger support from the infection data on this species as it provided a better fit than both the snowball $(\mathrm{F}=10.92, P=0.001)$ and the linear model $(\mathrm{F}=5.25, P=0.014)$. The analysis of the residuals confirmed that the slowdown model predicts reproductive compatibility well $(P=0.985)$, although the run test was marginally significant $(P=0.047)$. This support is entirely consistent with the estimate of the parameter $a$ being higher than that obtained with the first infection data set, on S. latifolia.

Microbotryum F1 hybrid sterility and DNA content in F1 gametes. The inability of the gametes produced by Microbotryum F1 hybrids to undergo syngamy (Figure 5e) increases with genetic distance between the parents, as does DNA content per gamete (Figure 5f). For both data sets, the slowdown model converges towards the linear model as indicated by $\mathrm{F}$-tests $(\mathrm{F}=0.95, P=0.536$ and $\mathrm{F}=0.999, P=0.503)$, and small values of parameter $a$. Attempting to fit the snowball model does not provide better results than the linear one, neither for the first data set $(F=1.17, P=0.393)$ nor for the second one $(F=1.51$, 


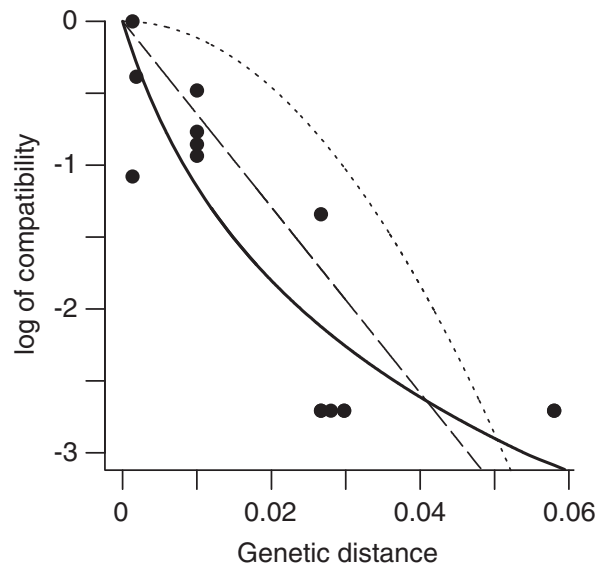

Figure 6 Evolution of Microbotryum (Basidiomycetes) F2 hybrids' inviability. Inviability is measured as the inability of Microbotryum F2 hybrids to infect host plants and produce symptoms. Continuous, broken and dotted lines stand for the 'slowdown', 'linear' and 'snowball' model, respectively. For each data set, the best model appears in bold.

$P=0.241)$. The convergence in the predictions of the three models is confirmed by the analysis of the residuals where the $P$-values obtained in each of the two non-parametric tests are very similar for all models ( $P=0.190,0.088,0.088$, and $P=0.846,0.980,0.846$ for the two tests on the sterility data set, that is inability to undergo syngamy; $P=0.898,0.852,0.852$, and $P=0.078,0.452,0.452$ for the two tests on DNA content). There is thus no departure from a constant rate of decrease in compatibility between species.

As the same (linear) models were selected for both hybrid inviability and sterility in Microbotryum, their parameters can be compared, in particular the $e$ parameters, corresponding to the speed of compatibility decrease. These parameters differ substantially between the two data sets, by about two orders of magnitude, showing that hybrid inviability decreases much more rapidly in Microbotryum than hybrid sterility (1985 and 8773 vs 45.5 and 33.9 in Table 1; see also Figure 5).

Microbotryum F2 hybrid inviability. Regarding the inability of Microbotryum F2 hybrids to infect host plants, the slowdown and the linear models provide better fits than the snowball model (F=5.37, $P=0.002$ and $\mathrm{F}=2.73, P=0.039)$, but none of them provide a better fit than the other $(\mathrm{F}=1.82, P=0.143)$. The quality of the fit obtained from these two models is also supported by the large $P$-values in the analysis of the distribution of their residuals $(P=0.779$ and 0.970 for the slowdown; $P=0.594$ and 0.643 for the slowdown). As for F1 hybrid sterility and DNA content in F1 gametes, there is no evidence that the rate of loss of compatibility increases with genetic distance (Figure 6).

\section{DISCUSSION}

The comparison between models of the evolution of reproductive isolation with genetic distance using data sets on fungi provides support for a slowdown model in the presyngamy compatibility among basidiomycetes, but not among ascomycetes. Regarding the evolution of postsyngamy isolation, we found no evidence for a snowball model in either ascomycetes or the anther smut species complex, but instead a linear or sublinear evolution of incompatibility.

\section{Reinforcement on presyngamy compatibility in fungi}

Our approach provided a clear support for both the slowdown model and a faster decrease of reproductive compatibility in sympatry than in allopatry for the data set on presyngamy reproductive isolation in basidiomycetes. The co-occurrence of these patterns had already been detected in starfish and drosophila (Gourbière and Mallet, 2010), and altogether they can be interpreted as the existence of reinforcement of reproductive isolation in sympatry. That a slowdown pattern suggests a process of reinforcement possibly relies on the assumptions we used to map theories for the accumulation of incompatibilities onto reproductive isolation; all incompatibilities have an identical intrinsic deleterious effect (although their absolute effect decline in the multiplicative fitness scheme considered) and there is no epistasis between the fitness effect of these incompatibilities (see pages 5, 17 and 18 in Gourbiere and Mallet (2010), for an extensive discussion of those assumptions). Alternative models could be proposed, assuming heterogeneous fitness effect of incompatibilities, admixture of epistatic and non-epistatic incompatibilities, or epistasis in fitness. Although these more complex models could possibly provide a better fit to the data, evidences for these alternative assumptions are so disparate (for example, spread across different species) that it would be difficult to propose a credible model for any given taxa. Our results confirm the previous conclusion of Le Gac and Giraud (2008) about the occurrence of reinforcement in fungi that were based on mere comparison of reproductive compatibility patterns in those same data sets. This study significantly strengthens these earlier conclusions by fitting the data to quantitative predictions made by three different theories on the evolution of reproductive isolation. This effectively allows concluding that the pattern is consistent with reinforcement, and that reinforcement is the best explanation when compared with the two theoretical alternatives that are discussed in the literature.

The slowdown model also provided the best fit for the evolution of presyngamy gamete compatibility in the anther smut fungi, which we also interpreted as suggesting the occurrence of reinforcement in basidiomycetes (but see discussion above). The percentage of gametes undergoing syngamy with gametes of another species indeed decreased rapidly at the lowest genetic distances, which was not detected in a previous study (Le Gac et al., 2007b). Surprisingly, however, the degree of syngamy compatibility did not continue to decrease at larger genetic distances, remaining quite high. That inhibition of interspecific gamete fusion does not further decrease at higher genetic distance is intriguing given the evolution of postsyngamy isolation, with continual increase of hybrid inviability and sterility at these genetic distances, and the overlapping ranges of the species (Bucheli et al., 2000; Le Gac et al., 2007a, b; Hood et al., 2010; Gladieux et al., 2010b; Vercken et al., 2010). One may think that reinforcement would have kept on occurring to avoid producing unfit hybrids, especially at the greatest genetic distances where postsyngamy isolation is the strongest. It may be that, reinforcement occurring only within sympatric populations, it could not be detected with this data set including allopatric populations. In contrast to this view, however, a previous experiment has shown that the propensity of conjugation was not lower between heterospecifics than conspecifics even between individuals occurring within the very same populations (Refrégier et al., 2010). The explanation for the persistence of high degree of syngamy compatibility at large genetic distance may instead be that the strength of selection for increasing assortative mating via mate choice may be too low in Microbotryum, because hybridization is sufficiently rare in nature. The cumulative effects of other presyngamy barriers, such as differences in habitat and pollinator of the plant 
species (Goulson and Jerrim, 1997; van Putten et al., 2007; Gladieux et al., 2010b) and high rates of selfing (>0.90; Giraud et al., 2005, 2008b; Hood and Antonovics, 2000; Gladieux et al., 2010b), may be strong enough such that very few hybrids are produced in nature even with weak preference for conspecifics (Bucheli et al., 2000; Refrégier et al., 2010; Gladieux et al., 2010b; Gibson et al., 2012).

In contrast to basidiomycetes, ascomycetes did not show a slowdown tendency in the evolution of reproductive isolation. There was no support in ascomycetes for any of the three models over the others, neither in the sympatric nor the allopatric data sets. Inability to reject the linear model can be interpreted as a lack of a strong reinforcement. In fact, many ascomycete sympatric species pairs show complete interfertility, even in sympatry and at the highest genetic distances for which data on experimental crosses were available. Barriers to gene flow other than intrinsic postzygotic isolation or premating isolation in the form of mate choice are thus probably maintaining the integrity of these closely related species pairs in sympatry. As ascomycetes mate within the substrate (often a host plant) where they have first been able to persist and grow, it has been proposed that substrate/host adaptation could pleiotropically yield reproductive isolation: only the individuals able to grow in the same habitat/host can mate, so that adaptation automatically leads to reproductive isolation (Giraud et al., 2006, 2008a, 2010; Gladieux et al., 2011; Servedio et al., 2011). Closely related species of ascomycetes specialized onto different ecological niches would thus not require active mate choice to remain genetically differentiated in sympatry, as their niche adaptation would pleiotropically restrict gene flow (Giraud et al., 2006, 2008a, 2010; Gladieux et al., 2011; Servedio et al., 2011). This stands in contrast to the case of basidiomycetes, where large-scale dispersal occurs before growing in their substrate. In basidiomycetes, mating can therefore occur even between individuals specialized on different substrates, so that mate choice would be needed for preventing gene flow between sympatric species, and reinforcement would be expected. In ascomycetes, in contrast, substrate adaptation pleiotropically induces assortative mating, as only individuals able to mate within the same substrate can mate, so that no further intrinsic reinforcement on mate choice is expected. Interestingly, the single documented case of reinforcement in ascomycetes occurred in non-pathogenic species growing in the same type of substrate (partially burnt vegetation): Turner et al. (2010) reported that isolation between Neurospora crassa and Neurospora intermedia was stronger between sympatric populations than allopatric populations. Notably, reinforced isolation involved a postsyngamy barrier, abortion of fruiting bodies, which might be adaptive as it increased the likelihood that individuals successfully mate conspecifically after maladaptive hybrid fertilization (Turner et al., 2011).

The fact that many ascomycete sympatric species pairs showed interfertility, even in sympatry and at high genetic distances, raises the problem of species criteria. The sibling species included in the present data sets have been recognized as distinct mostly based on gene genealogies showing that they do not exchange genes for sufficient time as to show complete lineage sorting (that is, the criterion of concordance of multiple gene genealogies). That such sibling species pairs retain interfertility in vitro long after speciation, even in sympatry, shows that this criterion is not always the most suitable to delimit species, and should not be considered as absolutely required (Baum and Shaw, 1995; de Queiroz, 1998; Giraud et al., 2008a; Gladieux et al., 2010a; Cai et al., 2011). In fact, the most finely discriminating species criterion in fungi, and the currently most widely used one, is precisely the criterion of concordance between multiple gene genealogies, that directly assess the occurrence of recombination, or lack of thereof (Giraud et al., 2008a; Taylor et al., 1999; Gladieux et al., 2010a; Cai et al., 2011).

\section{Lack of evidence for the 'snowball' in fungi}

Overall, we did not find evidence that hybrid inviability and sterility 'snowballed' with genetic distance either in ascomycetes or at any stage of the Microbotryum life cycle. The absence of significant departure from a linear decrease of the log of compatibility with genetic distance for early hybrid inviability of Microbotryum measured as hyphal growth is thus not consistent with the expected pattern that would be caused by the accumulation of DM incompatibilities of similar (non-epistatic) effects on hybrid fitness. We, however, note that the negative value of the slowdown model's parameter $a$ and the sign of the deviations from linearity do suggest a slower than linear rate of substitution at low genetic distance. Failing to detect a clear 'snowball' pattern may just be caused by the lack of statistical power associated with the size of the data set. It has also been proposed that the 'missing snowball' (Johnson, 2006) in empirical studies may be explained by incompatibilities having diminishing return, which could cancel out the non-linear increase in their number (JMendelson et al., 2004; JTurelli and Moyle, 2007). Alternatively, hyphal growth of hybrids may be lowered by chromosomal rearrangements leading to heterozygote disadvantage (Walsh, 1982; BrittonDavidian et al., 2000; Louis, 2009), which indeed produces a linear evolution of the log of compatibility between taxa.

For the ability of Microbotryum hybrids to produce symptoms on plants, the slowdown models provided the best fits, both for F1 and F2 hybrids. This suggests that both F1 and F2 hybrid inviability in Microbotryum integrated over the whole life cycle is not mainly caused by the accumulation of DM incompatibilities with similar fitness effects, as these are expected to produce a snowball pattern (but see discussion above). Instead, hybrid inviability increases rapidly and then slows down before reaching complete inability to infect plants. Because the fitness of the hybrids is measured as the ability to infect plants, this may represent mostly ecologically based inviability with a simpler genetic basis than DM epistatic incompatibilities. In particular, this pattern is potentially consistent with the mechanisms of interaction between pathogens and their host plants: plants carry resistance genes in their genome, producing proteins recognizing pathogens, recognition triggering a defense reaction from the plant that impairs tissue colonization by the pathogen. Pathogens in turn evolve to avoid recognition by their host plant (Salvaudon et al., 2008). Specialized pathogens often cannot colonize non-host plants because these plants are able to recognize their presence and block infection (de Wit et al., 2009). This simple genetic basis of the infection ability involving a few genes with major effects and no epistasis may produce a pattern of slowdown inviability. Ecologically dependent hybrid inviability has been poorly explored in pathogenic fungi so far, not mentioning their genetic basis. The existence of ecologically dependent hybrid inviability has nevertheless been reported in several taxa (Coyne and Orr, 2004, chap 7; Egan and Funk, 2009; Schluter, 2009).

The evolution of sterility in Microbotryum did not fit a 'snowball' pattern either. Both the inability of the gametes produced by Microbotryum F1 hybrids to undergo syngamy and DNA content per gamete increased linearly with genetic distance between the parents. Again, this pattern could hypothetically be caused by an accumulation of incompatibilities with epistatic effects decreasing with their number, exactly cancelling out the increase in number. The linear pattern is alternatively consistent with an impact of chromosomal rearrangements that likely leads to simple heterozygote 
disadvantage (Coyne and Orr, 2004; Gavrilets, 2004; Welch, 2004). In fact, different Microbotryum species exhibit highly divergent karyotypes, even between closely related species (Hood et al., 2003). The gamete sterility is thus most likely due to karyotypes differences between Microbotryum species, resulting in the observed abnormal genome content in the gametes. Such a role of chromosomal rearrangements in fungal hybrid sterility has previously been suggested between Saccharomyces paradoxus and Saccharomyces cariocanus, which differ by four large reciprocal translocations but otherwise diverge very little in DNA sequence (Liti et al., 2006; Greig, 2009). An elegant study using genetic engineering showed that reconfiguring the $S$. cerevisae genome to make it collinear to the Saccharomyces mitakae genome allowed the generation of interspecific hybrids with higher fertility (Delneri et al., 2003).

The overall lack of unequivocal support for the snowball model in postsyngamy compatibility is consistent with a previous study on the evolution of reproductive isolation in other eukaryotes, and in particular in the yeast Saccharomyces (Gourbière and Mallet, 2010). Fungi show high genomic fluidity that may often yield sterility linked to chromosomal rearrangements (Zolan, 1995), either due to problems of non-disjunction at meiosis or epigenetic mechanisms that are involved in defending the fungal genomes against selfish DNA elements (Shiu et al., 2001; Greig et al., 2003; Matzke and Birchler, 2005). For instance, the genome defense against transposable elements known as repeat-induced point mutation (Galagan and Selker, 2004) may be expected to act upon the segmental duplications and aneuploidy in hybrids. None of these processes include epistatic effect on fitness, and they represent alternative hypotheses to the accumulation of DM incompatibilities, that could potentially contribute in explaining the evolution of reproductive isolation in fungi. Several genome-wide analyses have failed to find any DM incompatibilities (Greig, 2007; Lee et al., 2008; Greig, 2009; Kao et al., 2010), reinforcing the view that they may not be the most common cause of hybrid inviability and sterility in fungi.

Our results, however, do not mean that epistatic incompatibilities do not exist in fungi or do not have a role in reproductive isolation in this group. In fact, a number of epistatic incompatibilities have been identified in diverse fungi, several involving mtDNA (Lee et al., 2008; Anderson et al., 2010; Chou et al., 2010; Dettman et al., 2010). Their fitness consequences may be hidden in our data sets by other causes with stronger and linear effects, such as chromosomal rearrangements or ecologically based mechanisms. As highlighted above, it is also possible that the level of epistasis in fitness changes with the accumulation of DM incompatibilities in a way that creates a more linear evolution of reproductive isolation. More studies are needed to assess how the number of genetic incompatibilities accumulating with time in fungi and their epistatic effects on hybrid fitness. Interestingly, two recent studies looking for the first time at the evolution of the number of incompatibilities with genetic distance, and not only decrease in fitness, supported the snowball model, respectively, in Drosophila (Matute et al., 2010) and tomatoes (Moyle and Nakazato, 2010). The number of points to fit the curves was, however, still limited. More functional studies are definitely needed to identify the genetic mechanisms underlying hybrid inviability and sterility in fungi.

Interestingly, inviability appeared to evolve faster than sterility in Microbotryum, which is the opposite of the pattern generally found in plants and animals (Presgraves, 2008). Some of the reasons invoked for a more rapid evolution of sterility in plants and animals are asymmetry in the degree of heterozygosity of sex chromosomes and sexual selection, both of which do not exist in fungi. Mating type chromosomes in fungi are indeed always heterozygous, as haploid cells have to carry different mating types to mate (Billiard et al., 2011) and sexual selection is virtually absent, as few phenotypic traits exist that could be targets of such selection.

\section{CONCLUSION}

This study on the evolution of reproductive isolation in fungi confirms that fungi are tractable models for studying speciation and for inferring generalities concerning the evolution of reproductive isolation. The analyses support the occurrence of reinforcement, as reported in plants and animals (Lukhtanov et al., 2005; Kay and Schemske, 2008), as well as the rarity of evidence for a snowball pattern in the evolution of reproductive isolation. More studies are, however, required in fungi to investigate the causes of hybrid inviability and sterility, in particular on the number of epistatic incompatibilities and on the existence of ecologically based hybrid inviability.

\section{DATA ARCHIVING}

There were no data to deposit.

\section{CONFLICT OF INTEREST}

The authors declare no conflict of interest.

\section{ACKNOWLEDGEMENTS}

We thank Amanda K Gibson, Pierre Gladieux, Mickaël Le Gac, Christophe Lemaire and three anonymous referees for comments on an earlier version of the paper. T Giraud acknowledges the ANR grants 06-BLAN-0201 and 07-BDIV-003 and S Gourbière the EU grant FP7-PEOPLE-2009-IEF 253483.

Anderson JB, Funt J, Thompson DA, Prabhu S, Socha A, Sirjusingh C et al. (2010). Determinants of divergent adaptation and Dobzhansky-Muller Interaction in experimental yeast populations. Curr Biol 20: 1-6.

Anderson PK, Cunningham AA, Patel NG, Morales FJ, Epstein PR, Daszak P (2004). Emerging infectious diseases of plants: pathogen pollution, climate change and agrotechnology drivers. Trends Ecol Evol 19: 535.

Baum DA, Shaw KL (1995). Genealogical perspectives on the species problem. In: Hoch PC, Stephenson AG (eds) Experimental and Molecular Approaches to Plant Biosystematics- Monographs in Systematic Botany from the Missouri Botanical Garden. Missouri Botanical Garden Press: St. Louis, MO, USA. Vol. 53, pp 289-303.

Billiard S, Lopez-Villavicencio M, Devier B, Hood ME, Fairhead C, Giraud T (2011). Having sex, yes, but with whom? Inferences from fungi on the evolution of anisogamy and mating types. Biol Rev 86: 421-442.

Bolnick DI, Near TJ (2005). Tempo of hybrid inviability in centrarchid fishes (Teleostei: Centrarchidae). Evolution 59: 1754-1767.

Britton-Davidian J, Catalan J, da Graca RM, Ganem G, Affray JC, Capela R et al. (2000). Rapid chromosomal evolution in mice. Nature 403: 158

Bucheli E, Gautschi B, Shykoff JA (2000). Host specific differentiation in the anther smut fungus Microbotryum violaceum as revealed by microsatellites. J Evol Biol 13: 188-198.

Cai L, Giraud T, Zhang N, Begerow D, Cai G, Shivas RG (2011). The evolution of species concepts and species recognition criteria in plant pathogenic fungi. Fungal Div 46: 121-131.

Chou J-Y, Hung Y-S, Lin K-H, Lee H-Y, Leu J-Y (2010). Multiple molecular mechanisms cause reproductive isolation between three yeast species. PLOS Biol 8: e1000432.

Coyne J, Orr HA (1989). Patterns of speciation in Drosophila. Evolution 43: 362-381.

Coyne J, Orr HA (1997). 'Patterns of speciation in Drosophila' revisited. Evolution 51: 295-303.

Coyne JA, Orr HA (2004). Speciation. Sinauer Associates: Sunderland, MA.

de Queiroz K (1998). The general lineage concept of species, and the process of speciation. In: Howard DJ, Berlocher SH (eds) Endless Forms: Species and Speciation. Oxford University Press: Oxford, pp 57-75.

de Vienne DM, Refrégier G, Hood ME, Guigue A, Devier B, Vercken E et al. (2009). Hybrid sterility and inviability in the parasitic fungal species complex. Microbotryum. J Evol Biol 22: 683-698.

de Wit P, Mehrabi R, van den Burg HA, Stergiopoulos I (2009). Fungal effector proteins: past, present and future. Mol Plant Pathol 10:6 735-747.

Delneri D, Colson I, Grammenoudi S, Roberts IN, Louis EJ, Oliver SG (2003). Engineering evolution to study speciation in yeasts. Nature 422: $68-72$. 
Dettman JR, Anderson JB, Kohn LM (2010). Genome-wide investigation of reproductive isolation in experimental lineages and natural species of Neurospora: identifying candidate regions by microarray-based genotyping and mapping. Evolution 64: 694-709.

Dettman JR, Sirjusingh C, Kohn LM, Anderson JB (2007). Incipient speciation by divergent adaptation and antagonistic epistasis in yeast. Nature 447: 585 .

Edmans S (2002). Does parental divergence predict reproductive compatibility? Trends Ecol Evol 17: 520-527.

Egan SP, Funk DJ (2009). Ecologically dependent postmating isolation between sympatric host forms of Neochlamisus bebbianae leaf beetles. Proc Natl Acad Sci USA 106: $19426-19431$.

Fitzpatrick BM (2002). Molecular correlates of reproductive isolation. Evolution 56 191-198.

Galagan J, Selker E (2004). RIP: the evolutionary cost of genome defense. Trends Ecol Ecol 20: 417-423.

Gavrilets S (2004). Fitness Landscapes and the Origin of Species. Princeton University Press: Princeton, NJ.

Gibson A, Hood M, Giraud T (2012). Sibling competition arena: selfing and a competition arena can combine to constitute a barrier to gene flow in sympatry. Evolution 954 $1-14$

Giraud T, Gladieux P, Gavrilets S (2010). Linking the emergence of fungal plant diseases with ecological speciation. Trends Ecol Ecol 25: 387-395.

Giraud T, Jonot O, Shykoff JA (2005). Selfing propensity under choice conditions in a parasitic fungus, Microbotryum violaceum, and parameters influencing infection success in artificial inoculations. Int J Plant Sci 166: 649-657.

Giraud T, Refrégier G, de Vienne DM, Le Gac M, Hood ME (2008a). Speciation in fungi. Fung Genet Biol 45: 791-802.

Giraud T, Villaréal L, Austerlitz F, Le Gac M, Lavigne C (2006). Importance of the life cycle in host race formation and sympatric speciation in parasites. Phytopathology 96 : 280-287.

Giraud T, Yockteng R, Lopez-Villavicencio M, Refrégier G, Hood ME (2008b). The mating system of the anther smut fungus, Microbotryum violaceum: selfing under heterothallism. Euk Cell 7: 765-775.

Gladieux P, Byrnes E, Fisher M, Aguileta G, Heitman J, Giraud T (2010a). Epidemiology and evolution of fungal pathogens, in plants and animals. In: Tibayrenc $M$ (ed.) Genetics and Evolution of Infectious Diseases. Elsevier: Burlington, MA

Gladieux P, Guérin F, Giraud T, Caffier V, Lemaire C, Parisi L et al. (2011). Emergence of novel fungal pathogens by ecological speciation: importance of the reduced viability of immigrants. Mol Ecol 20: 4521-4532.

Gladieux P, Vercken E, Fontaine MC, Hood ME, Jonot O, Couloux A et al. (2010b). Maintenance of fungal pathogen species that are specialized to different hosts: allopatric divergence and introgression through secondary contact. $\mathrm{Mol} \mathrm{Biol} \mathrm{Evol} \mathrm{28}$ 459-471.

Goulson D, Jerrim K (1997). Maintenance of the species boundary between Silene dioica and S. latifolia (red and white campion). Oikos 79: 115-126.

Gourbière S, Mallet J (2010). Are species real? The shape of the species boundaries with exponential failure, reinforcement and the 'missing snowball'. Evolution 64: 1-24.

Greig D (2007). A screen for recessive speciation genes expressed in the gametes of $F$ hybrid yeast. Plos Genetics 3: 281-286.

Greig D (2009). Reproductive isolation in Saccharomyces. Heredity 102: 39-44

Greig D, Travisano M, Louis EJ, Borts RH (2003). A role for the mismatch repair system during incipient speciation in Saccharomyces. J Evol Biol 16: 429-437.

Hood ME, Antonovics J (2000). Intratetrad mating, heterozygosity, and the maintenance of deleterious alleles in Microbotryum violaceum (=Ustilago violacea). Heredity $\mathbf{8 5}$ 231-241.

Hood ME, Antonovics J, Heishman H (2003). Karyotypic similarity identifies multiple host-shifts of a pathogenic fungus in natural populations. Infect Genet Evol 2: 167 172.

Hood ME, Mena-Alí JI, Gibson AK, Oxelman B, Giraud T, Yockteng R et al. (2010). Distribution of the anther-smut pathogen Microbotryum on species of the Caryophyllaceae assessed from natural history collections. New Phytol 187: 217-229.

Johnson NA (2006). Patterns and processes of speciation: the evolution of reproductive isolating barriers. In: Fox CW, Wolf JB (eds) Evolutionary genetics. Concepts and Case Studies. Oxford University Press: Oxford, pp 374-386.

Kao KC, Schwartz K, Sherlock G (2010). A genome-wide analysis reveals no nuclear Dobzhansky-Muller pairs of determinants of speciation between $S$. cerevisiae and S. paradoxus, but suggests more complex incompatibilities. PLoS Genet 6 : e1001038.

Kay KM, Schemske DW (2008). Natural selection reinforces speciation in a radiation of neotropical rainforest plants. Evolution 62: 2628-2642.

Knowlton N, Weigt LA, Solorzano LA, Mills DK, Bermingham E (1993). Divergence in proteins, mitochondrial DNA and reproductive compatibility across the Isthmus of Panama. Science 260: 1629-1632.

Le Gac M, Giraud T (2008). Existence of a pattern of reproductive character displacement in Basidiomycota but not in Ascomycota. J Evol Biol 21: 761-772.
Le Gac M, Hood ME, Fournier E, Giraud T (2007a). Phylogenetic evidence of host-specific cryptic species in the anther smut fungus. Evolution 61: 15-26.

Le Gac M, Hood ME, Giraud T (2007b). Evolution of reproductive isolation within a parasitic fungal complex. Evolution 61: 1781-1787.

Lee H-Y, Chou J-Y, Cheong L, Chang N-H, Yang S-Y, Leu1 J-Y (2008). Incompatibility of nuclear and mitochondrial genomes causes hybrid sterility between two yeast species. Cell 135: 1065-1073.

Liti G, Barton DBH, Louis EJ (2006). Sequence diversity, reproductive isolation and species concepts in Saccharomyces. Genetics 174: 839-850.

Louis EJ (2009). Evolutionary genetics: origins of reproductive isolation. Nature 457 $549-550$

Lukhtanov VA, Kandul NP, Plotkin JB, Dantchenko AV, Haig D, Pierce NE (2005). Reinforcement of pre-zygotic isolation and karyotype evolution in Agrodiaetus butterflies. Nature 436: 385-389.

Matute DR, Butler IA, Turissini DA, Coyne JA (2010). A test of the snowball theory for the rate of evolution of hybrid incompatibilities. Science 329: 1518-1521.

Matzke M, Birchler J (2005). RNAi-mediated pathways in the nucleus. Nat Rev Genet 6 : 24-35.

Mendelson TC, Inouye BD, Rausher MD (2004). Quantifying patterns in the evolution of reproductive isolation. Evolution 58: 1424-1433.

Moyle LC, Nakazato T (2010). Hybrid incompatibility 'snowballs' between Solanum species. Science 329: 1521

Orr HA (1995). The population genetics of speciation: the evolution of hybrid incompatibilities. Genetics 139: 1805-1813.

Orr HA, Turelli M (2001). The evolution of postzygotic isolation: accumulating Dobzhansky-Muller incompatibilities. Evolution 55: 1085-1094.

Presgraves D (2008). Sex chromosomes and speciation in Drosophila. Trends Genet 24: $336-343$.

Presgraves DC (2002). Patterns of post-zygotic isolation in Lepidoptera. Evolution 56 1168-1183.

Refrégier G, Hood ME, Giraud T (2010). No evidence of reproductive character displacement between two sister fungal species causing anther smut disease in Silene. Int J Plant Sci 171: 847-859.

Refrégier G, Le Gac M, Jabbour F, Widmer A, Hood ME, Yockteng R et al. (2008). Cophylogeny of the anther smut fungi and their Caryophyllaceous hosts: prevalence of host shifts and Importance of delimiting parasite species. BMC Evol Biol 8: 100

Salvaudon L, Giraud T, Shykoff JA (2008). Genetic diversity in natural populations: a fundamental component of plant-microbe interactions. Curr Opin Plant Biol 11 135-143.

Sasa MM, Chippindale PT, Johnson NA (1998). Patterns of postzygotic isolation in frogs Evolution 52: 1811-1820.

Schluter D (2009). Evidence for ecological speciation and its alternative. Science 323 : 737-741

Servedio MR, Doorn GSV, Kopp M, Frame AM, Nosil P (2011). Magic traits in speciation: 'magic' but not rare? Trends Evol Evol 26: 389-397.

Shiu PKT, Raju NB, Zickler D, Metzenberg RL (2001). Meiotic silencing by unpaired DNA Cell 107: 905-916

Stajich JE, Berbee ML, Blackwell M, Hibbett DS, James TY, Spatafora JW et al. (2009). The Fungi. Curr Biol 19: R840-R845.

Taylor JW, Geiser DM, Burt A, Koufopanou V (1999). The evolutionary biology and population genetics underlying fungal strain typing. Clin Microbiol Rev 12: 126-146.

Turelli M, Moyle LG (2007). Asymmetric postmating isolation: Darwin's corollary to Haldane's Rule. Genetics 176: 1059-1088.

Turner E, Jacobson DJ, Taylor JW (2010). Reinforced postmating reproductive isolation barriers in Neurospora, an Ascomycete microfungus. J Evol Biol 23: 1642-1656.

Turner E, Jacobson DJ, Taylor JW (2011). Genetic architecture of a reinforced, postmating, reproductive isolation barrier between Neurospora species indicates evolution via natural selection. PLoS Genet 7: e1002204.

van Putten WF, Elzinga JA, Biere A (2007). Host fidelity of the pollinator guilds of Silene dioica and Silene latifolia: Possible consequences for sympatric host race differentiation of a vectored plant disease. Int J Plant Sci 168: 421-434.

Vercken E, Fontaine MC, Gladieux P, Hood ME, Jonot O, Giraud T (2010). Glacial refugia in pathogens: European genetic structure of anther smut pathogens on Silene latifolia and S. dioica. PloS Pathog 6: e1001229.

Walsh JB (1982). Rate of accumulation of reproductive isolation by chromosome rearrangements. Am Nat 120: 510-532.

Weins J, Engstrom TN, Chippindale C (2006). Rapid diversification, incomplete isolation, and the 'speciation clock' in North American salamanders (Genus Plethodon): testing the hybrid swarm hypothesis of rapid radiation. Evolution 60: 2585-2603.

Welch JJ (2004). Accumulating Dobzhansky-Muller incompatibilities: reconciling theory and data. Evolution 58: 1145-1156.

Widmer A, Lexer C, Cozzolino S (2009). Evolution of reproductive isolation in plants. Heredity 102: 31-38.

Zolan ME (1995). Chromosome-length polymorphism in fungi. Microbiol Rev 59: 686-698. 